\title{
A Comparison of Vibroacoustic Response of Isotropic Plate with Attached Discrete Patches and Point Masses Having Different Thickness Variation with Different Taper Ratios
}

\author{
Bipin Kumar, ${ }^{1}$ Vinayak Ranjan, ${ }^{1}$ Mohammad Sikandar Azam, ${ }^{1}$ Piyush Pratap Singh, \\ Pawan Mishra, ${ }^{1}$ K. Priya Ajit, ${ }^{2}$ and Praveen Kumar ${ }^{1}$ \\ ${ }^{1}$ Department of Mechanical Engineering, Indian School of Mines, Dhanbad, Jharkhand 826004, India \\ ${ }^{2}$ Department of Mining Machinery Engineering, Indian School of Mines, Dhanbad, Jharkhand 826004, India
}

Correspondence should be addressed to Bipin Kumar; bipinkbit@gmail.com

Received 24 June 2015; Revised 15 September 2015; Accepted 16 September 2015

Academic Editor: Sergio De Rosa

Copyright (c) 2016 Bipin Kumar et al. This is an open access article distributed under the Creative Commons Attribution License, which permits unrestricted use, distribution, and reproduction in any medium, provided the original work is properly cited.

A comparison of sound radiation behavior of plate in air medium with attached discrete patches/point masses having different thickness variations with different taper ratio of $0.3,0.6$, and 0.9 is analysed. Finite element method is used to find the vibration characteristics while Rayleigh integral is used to predict the sound radiation characteristics. Minimum peak sound power level obtained is at a taper ratio of 0.6 with parabolic increasing-decreasing thickness variation for plate with four discrete patches. At higher taper ratio, linearly increasing-decreasing thickness variation is another alternative for minimum peak sound power level suppression with discrete patches. It is found that, in low frequency range, average radiation efficiency remains almost the same, but near first peak, four patches or four point masses cause increase in average radiation efficiency; that is, redistribution of point masses/patches does have effect on average radiation efficiency at a given taper ratio.

\section{Introduction}

Vibration and sound radiation problems of plates with and without attached discrete mass/patch are used in many engineering application. Generally, variable thickness is used to alter the resonant frequency and to reduce weight and size of structure. The stiffness is a mechanical property of the material. The thickness will influence the dynamic stiffness $(\mathrm{D}=\mathrm{EI})$ because the inertia will change. Several researchers investigated the free vibration analysis of plate with variable thickness without attached discrete mass/patch. Appl and Byers [1] were early researchers to analyse the natural frequency of a rectangular plate having linear thickness variation in one direction using analytical method. The natural frequencies and node patterns for clamped and simply supported square plate of linearly variable thickness were analysed by Raju [2] using experimental method. Kuttler and Sigillito [3] proposed an analytical method for finding lower and upper bounds for vibrational frequencies of clamped plate having linear tapers with general boundary conditions.
Roy and Ganesan [4] analysed the dynamic response of an isotropic square plate with linear and parabolic thickness variation in one direction. Huang et al. [5] used a discrete method for finding the free vibration problem of orthotropic rectangular plate with variable thickness.

Structural vibration of plate causes radiation of sound. The prediction of sound radiated from vibration of plate is important to control the noise generated from vibrating structure. Several researchers investigated sound radiation behavior of isotropic and composite plates with uniform thickness subjected to time varying harmonic excitation. Petrone et al. [6] investigated the acoustic power radiated by aluminium foam sandwich panels using Rayleigh integral approach and finite element is used to estimate the distribution of velocity over the panel surface. Franco et al. [7] presented the vibroacoustic performance of a panel plate with random stiffness. In this regard, finite element models of selected macroconfigurations of a given sandwich panel are analysed in terms of both mean square structural velocity and 
radiated acoustic power. Altintas [8] investigated the effect of mass based imperfections on behavior of linear vibrating plates near degenerate modes. The principal objective in this study was to investigate the special behaviors of linear vibrating plates with special parameters near degenerate modes. S. Li and X. Li [9] reported that distributed masses could significantly affect the acoustic radiation behavior of plates. Putra and Thompson [10] investigated the sound radiation from rectangular baffled and unbaffled plates. Jeyaraj [11] presented the acoustic behavior of an isotropic plate with varying thickness using FEM and BEM methods. Nelisse et al. [12] analysed the sound radiation from both baffled and unbaffled plate by Rayleigh-Ritz method. Atalla et al. [13] gave a numerical solution for sound radiation of an unbaffled plate by Kirchhoff-Helmholtz equation.

This paper presents a comparison of vibroacoustic response of isotropic plate with attached discrete patches and point masses having different thickness variation with different taper ratios studied. The effects of redistribution of discrete patch/mass on acoustic radiation behavior of plate with varying thickness having different taper ratios of $0.3,0.6$, and 0.9 in one direction are investigated.

\section{Modelling and Analysis Procedure}

2.1. Free Vibration Analysis of Plate. The strain energy of plate $U$ is given by [15]:

$$
U=\frac{1}{2}\{u\}^{T}[K]\{u\},
$$

where $[K]$ is the stiffness matrix and $\{u\}$ is the nodal displacement vector.

The kinetic energy for the plate is given by [15]:

$$
T=\frac{1}{2}\{\dot{u}\}^{T}[M]\{\dot{u}\},
$$

where $[M]$ is the mass matrix.

The natural frequency and mode shape of the plate are obtained by solving the following eigenvalue problem for $\omega^{2}$ :

$$
\left([K]-\omega^{2}[M]\right) \varphi_{m n}=0,
$$

where $\varphi_{m n}$ is the mode shape function and $\omega$ is the natural frequency of plate.

\subsection{Acoustic Radiation Field of Rectangular Thin Plate due} to Flexural Vibration. It is assumed that the rectangular thin plate, simply supported along four edges in flexural vibration, is set on a flat rigid baffle of infinite extent as shown in Figure 1. The complex acoustics pressure amplitude $P(r)$ at position $r(R, \theta, \emptyset)$ can be written in terms of the plate surface velocity using the Rayleigh integral [16] evaluated over the plate surface given by

$$
P(r)=\frac{j k \rho c}{2 \pi} \int V_{X} \cdot \frac{e^{-j k R^{\prime}}}{R^{\prime}} d X,
$$

where $V_{X}$ is complex velocity amplitude normal to the surface at location $X=(x, y), k$ is acoustics wave number, $\rho$ is density

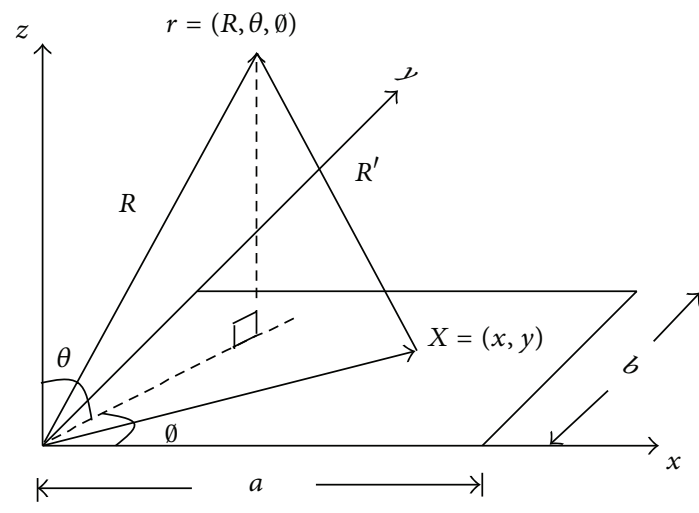

FIGURE 1: Coordinate system of a vibrating plate [14].

of air, $c$ is speed of sound, $R^{\prime}=|r-X|$, distance from the location on the plate to the observation point, and $R$ is radius of sphere. The term $e^{-j k R^{\prime}} / R^{\prime}$ is Half space Green's function [17].

By integrating the far-field acoustic intensity over a hemisphere of radius $R$, the total acoustic power [14] is given by

$$
W=\int_{0}^{2 \pi} \int_{0}^{\pi / 2} \frac{|p(r)|^{2}}{2 \rho c} R^{2} \sin \theta \cdot d \theta \cdot d \emptyset .
$$

The velocity $V(x, y)$ at any location $X$ on the plate can be found by summing over all the modes of structure vibration of plate [17] and is given by

$$
V(x, y)=\sum_{m=1}^{\infty} \sum_{n=1}^{\infty} u_{m n} \varphi_{m n}(x, y),
$$

where $u_{m n}$ is complex velocity amplitude of mode $(m, n)$ and $\varphi_{m n}$ is mode shape function at $(x, y)$.

For the plate with simply supported edges, the mode shape function $\varphi_{m n}(x, y)[17]$ is given by

$$
\varphi_{m n}(x, y)=\sin \left(\frac{m \pi x}{a}\right) \sin \left(\frac{n \pi y}{b}\right),
$$

where $a$ and $b$ are length and width of the plate.

The modal velocity amplitude $u_{m n}$ due to a point force applied at location $(x, y)[18]$ is given by

$$
u_{m n}=\frac{j \omega F \varphi_{m n}(x, y)}{\left[\omega_{m n}^{2}(1+j \eta)-\omega^{2}\right] M_{m n}},
$$

where $F$ is force amplitude, $\omega_{m n}$ is natural frequency, $\eta$ is damping loss factor, and $M_{m n}$ is modal mass.

The modal mass for the simply supported boundary [10] is given by

$$
M_{m n}=\int \rho_{p} h_{p} \varphi_{m n}^{2}(x, y) \cdot d s=\frac{M}{4},
$$

where $M$ is mass of plate, $\rho_{p}$ is density of plate, and $h_{p}$ is thickness of plate. 


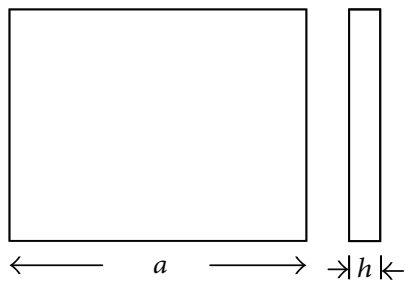

(a)

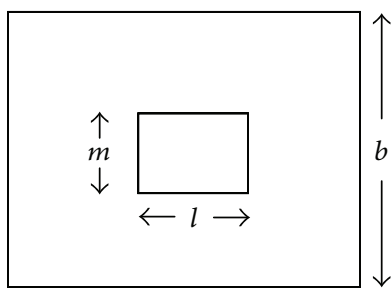

(b)

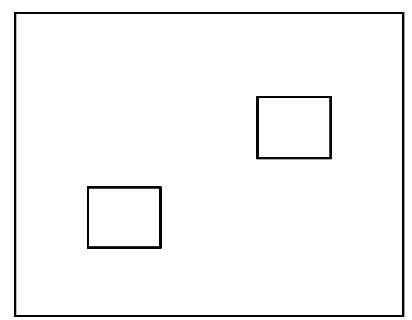

(c)

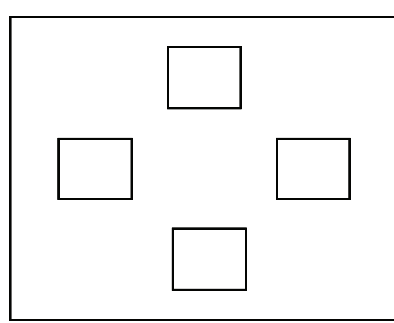

(d)

Figure 2: (a) Plate without any discrete patch/point mass, (b) plate with single patch, mass of patch $=0.1$ times plate mass, (c) plate with two discrete patches, mass of each patch $=0.05$ times plate mass, and (d) plate with four discrete patches, mass of each patch $=0.025$ times plate mass.

The natural frequency $\omega_{m n}[10]$ is given by

$$
\omega_{m n}=\left(\frac{B}{\rho_{p} h_{p}}\right)^{1 / 2}\left[\left(\frac{m \pi}{a}\right)^{2}+\left(\frac{n \pi}{b}\right)^{2}\right] \text {, }
$$

where $B=E h_{p}^{3} / 12\left(1-\mu^{2}\right)$ bending stiffness of plate.

By substituting (6) into (4), the sound pressure is given by

$$
\begin{aligned}
& P(r)=\sum_{m=1}^{\infty} \sum_{n=1}^{\infty} u_{m n}\left\{\frac{j k \rho c}{2 \pi} \int \varphi_{m n}(x, y) \cdot \frac{e^{-j k R^{\prime}}}{R^{\prime}} d s\right\} \\
& \text { Or, } P(r)=\sum_{m=1}^{\infty} \sum_{n=1}^{\infty} u_{m n} A_{m n}(r),
\end{aligned}
$$

where $A_{m n}(r)=(j k \rho c / 2 \pi) \int \varphi_{m n}(x, y) \cdot\left(e^{-j k R^{\prime}} / R^{\prime}\right) d s$.

An analytical solution of $A_{m n}[19]$ is given by

$$
A_{m n}(r)=\frac{j k \rho c}{2 \pi}\left(\frac{e^{-j k R}}{R}\right) \Psi,
$$

where

$$
\begin{array}{r}
\Psi=\frac{a b}{\pi^{2} m n}\left[\frac{(-1)^{m} e^{j \alpha}-1}{(\alpha / m \pi)^{2}-1}\right]\left[\frac{(-1)^{n} e^{j \beta}-1}{(\beta / n \pi)^{2}-1}\right], \\
\alpha=k a \sin \theta \cdot \cos \emptyset, \beta=k b \sin \theta \cdot \sin \emptyset .
\end{array}
$$

Since $P \cdot P^{*}=|P|^{2}$, substituting (12) into (5), the total acoustic power is given by

$$
\begin{aligned}
W= & \sum_{m=1}^{\infty} \sum_{n=1}^{\infty} \sum_{m^{\prime}=1}^{\infty} \sum_{n^{\prime}=1}^{\infty} u_{m n} u_{m^{\prime} n^{\prime}}^{*} \\
& \cdot \int_{0}^{2 \pi} \int_{0}^{\pi / 2} \frac{A_{m n}(r) \cdot A_{m^{\prime} n^{\prime}}^{*}(r)}{2 \rho c} R^{2} \sin \theta d \theta d \emptyset
\end{aligned}
$$

where $m^{\prime}$ and $n^{\prime}$ denote the value of $m$ and $n$ in conjugate form.

The radiation efficiency " $\sigma$ " [9] is given by

$$
\sigma=\frac{W}{\rho c s_{0}\left\langle\bar{V}^{2}\right\rangle}
$$

where $W$ is the radiated sound power, $\rho$ is density of medium, $c$ is the speed of sound in medium, $s_{0}$ is the total surface area of the structure, and $\left\langle\bar{V}^{2}\right\rangle$ is space average of the square of the velocity of the plate. In this paper, eigenvalue, sound power, and average radiation efficiency radiated from a plate having varying thickness (different taper ratios $0.3,0.6$, and 0.9 ) with discrete patches and point masses are analysed. Two arrangements of plate with attached discrete patches and point masses are considered. Plates with patches are shown in Figures 2(b)-2(d). Plates with point masses are similar to those of Figures 2(b)-2(d), when patches are replaced by equivalent point masses. The selection of point mass and patch is such that mass of "plate + patch" is equal to "plate + point mass." The dimensions and the physical properties of plate and patch are reported in Table 1. The thickness of plate is kept equal to $0.003 \mathrm{~m}$ for all the cases. The thickness of patch varies as reported in Table 1. The mass of plate without patches $/$ masses is $4.061 \mathrm{~kg}$. The mass of plate with one patch/one point mass or two patches/two point masses or four patches/four point masses remains the same and it is $4.455 \mathrm{~kg}$. Finite element method (FEM) has been used for vibration analysis and Rayleigh integral for sound power calculation. For modeling of plate, solid element 186 is used in ANSYS. A mild steel simply supported rectangular isotropic plate is used and excited by a concentrated force of amplitude $1 \mathrm{~N}$ applied at the center of the plate. The plate is assumed to be vibrating in air with air density $\rho=1.21 \mathrm{~kg} / \mathrm{m}^{3}$. The speed of sound " $c$ " of air is taken equal to $343 \mathrm{~m} / \mathrm{s}$, that is, the value at temperature of $20^{\circ}$. A structural damping coefficient of 0.01 is used for plate in air medium. For modeling the surrounding acoustic medium around the plate, FLUID30 and FLUID130 elements are used in ANSYS. FLUID30 is used to activate the fluid-structure interaction. The FLUID 130 elements are created on the surface of the outer sphere. This element simulates a condition of infinite space around the source and thus eliminates the error due to reflection of sound waves back to the source. The numbers of elements and nodes created are approximately 40000 and 13000, respectively, after proper convergence of results for different cases.

2.3. Thickness Variation. Four different types of variation in thickness [15] of the plate are considered for analysis. The variation of the thickness is shown in Figure 3, where 
TABLE 1: The dimension and the physical properties of plate and patch.

\begin{tabular}{lcccc}
\hline Dimensions & $\begin{array}{c}\text { Plate without patches/masses } \\
\text { (Figure 2(a)) }\end{array}$ & $\begin{array}{c}\text { Single patch } \\
\text { (Figure 2(b)) }\end{array}$ & $\begin{array}{c}\text { Two patches } \\
\text { (Figure 2(c)) }\end{array}$ & $\begin{array}{c}\text { Four patches } \\
(\text { Figure 2(d)) }\end{array}$ \\
\hline Length $[\mathrm{m}]$ & $a=0.455$ & $l=0.11375$ & $l=0.07583$ & $l=0.05678$ \\
Width $[\mathrm{m}]$ & $b=0.379$ & $m=0.0631$ & $m=0.04737$ & $m=0.03158$ \\
Thickness $[\mathrm{m}]$ & $h=0.003$ & $n=0.007$ & $n=0.007$ & 7850 \\
Density $\left[\mathrm{kg} / \mathrm{m}^{3}\right]$ & 7850 & 7850 & $2.10 E+11$ & 7850 \\
Young's modulus $\left[\mathrm{N} / \mathrm{m}^{2}\right]$ & $2.10 E+11$ & $2.10 E+11$ & 0.3 & $0.10 E+11$ \\
Poisson ratio & 0.3 & 0.3 & 0.3 \\
\hline
\end{tabular}

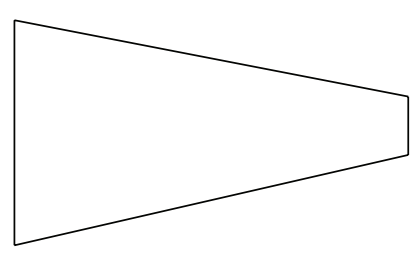

Case 1

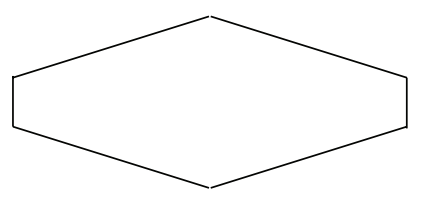

Case 3

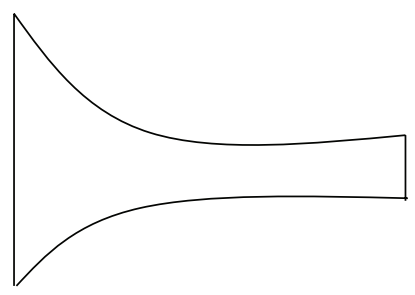

Case 2

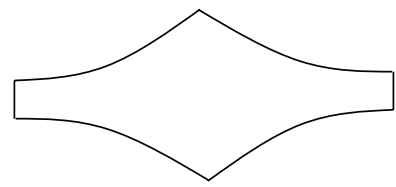

Case 4

FIGURE 3: Plate with different variable thickness [11].

thickness is varied linearly and linearly increasing-decreasing for cases 1 and 3 while thickness is varied parabolically and parabolically increasing-decreasing for cases 2 and 4 . In all four cases, mass of the plate and width of the plate are kept constant.

The equations of different types of thickness variation are given by [11]:

$$
\begin{aligned}
& h_{X}=h_{\max }\left\{1-H_{X}\left(\frac{x}{a}\right)^{n}\right\}, \\
& h_{X}=h_{\max }\left\{1-H_{X} \cdot \operatorname{abs}\left(1-\frac{2 x}{a}\right)^{n}\right\},
\end{aligned}
$$

where $h_{X}$ is the thickness of plate at $x$-distance, $n=1$ for linear profile and $n=2$ for parabolic profile, and $H_{X}$ is the taper parameter which is defined as $H_{X}=\left(1-h_{\min } / h_{\max }\right)$. Equation (17) represents decreasing thickness variation for linear and parabolic profile (cases 1 and 2). Equation (18) represents increasing-decreasing thickness variation for linear and parabolic profile (cases 3 and 4). The total volume of plate is constant which is given by

$$
\text { Volume }=h \cdot a \cdot b=b \cdot \int_{0}^{a} h_{X} d x \text {, }
$$

where $h$ is the thickness of uniform plate and $h_{X}$ is represented in terms of $h_{\max }$ and $H_{X}$. The taper parameters $H_{X}$ taken are $0.3,0.6$, and 0.9 .
TABLE 2: Comparison and validation of eigenfrequency $(\omega)$ in $\mathrm{Hz}$ of unloaded plate obtained in present work with that of S. Li and X. Li [9].

\begin{tabular}{lccc}
\hline Mode & (S. Li and X. Li [9]) & Present work & $\begin{array}{c}\text { \% variation in } \\
\text { natural } \\
\text { frequency }\end{array}$ \\
\hline$(1,1)$ & 87 & 86.989 & 0.012 \\
$(2,1)$ & 193 & 192.32 & 0.352 \\
$(1,2)$ & 241 & 240.81 & 0.078 \\
$(2,2)$ & 345 & 344.53 & 0.191 \\
\hline
\end{tabular}

\section{Result and Discussion}

3.1. Validation of Natural Frequency and Sound Power Calculation. For validation of natural frequency of a rectangular isotropic plate and sound power radiation, the published result of S. Li and X. Li [9] is considered as reported in Table 2 and Figure 4, respectively. From Figure 4 and Table 2, it is clear that the obtained results are in good agreement with the published result.

3.2. Free Vibration Analysis of Plate with Attached Discrete Patches/Point Masses with Different Taper Parameter $\left(H_{X}\right)$. The effects on natural frequencies due to attached point masses and discrete patches are investigated for different cases keeping the mass of the "plate + patches" and "plate + point mass" constant. 
TABLE 3: Comparison of first four eigenfrequencies $(\omega)$ in $\mathrm{Hz}$ of unloaded plate with attached discrete patches.

\begin{tabular}{|c|c|c|c|c|c|c|c|}
\hline Mode & $\begin{array}{c}\text { Unloaded } \\
\text { plate } \\
\text { (Figure } 2(\mathrm{a}) \text { ) }\end{array}$ & $\begin{array}{c}\text { Plate with } \\
\text { one discrete } \\
\text { patch } \\
\text { (Figure } 2(\mathrm{~b}))\end{array}$ & $\begin{array}{l}\% \text { variation } \\
\text { in natural } \\
\text { frequency }\end{array}$ & $\begin{array}{l}\text { Plate with } \\
\text { two discrete } \\
\text { patches } \\
\text { (Figure } 2(\mathrm{c}) \text { ) }\end{array}$ & $\begin{array}{l}\% \text { variation } \\
\text { in natural } \\
\text { frequency }\end{array}$ & $\begin{array}{c}\text { Plate with } \\
\text { four discrete } \\
\text { patches } \\
\text { (Figure } 2(\mathrm{~d}) \text { ) }\end{array}$ & $\begin{array}{c}\% \text { variation } \\
\text { in natural } \\
\text { frequency }\end{array}$ \\
\hline 1st & 87 & 86.942 & 0.066 & 85.865 & 1.304 & 85.102 & 2.181 \\
\hline 2nd & 193 & 192.74 & 0.134 & 191.84 & 0.601 & 191.19 & 0.937 \\
\hline $3 \mathrm{rd}$ & 241 & 239.43 & 0.651 & 237.88 & 1.294 & 235.43 & 2.311 \\
\hline 4 th & 345 & 341.47 & 1.023 & 340.54 & 1.292 & 337.57 & 2.153 \\
\hline
\end{tabular}

TABLE 4: Comparison of first four eigenfrequencies $(\omega)$ in $\mathrm{Hz}$ of unloaded plate with attached discrete point masses.

\begin{tabular}{lccccccc}
\hline Mode & $\begin{array}{c}\text { Unloaded } \\
\text { plate } \\
\text { (Figure 2(a)) }\end{array}$ & $\begin{array}{c}\text { Plate with } \\
\text { one discrete } \\
\text { point mass } \\
\text { (Figure 2(b)) }\end{array}$ & $\begin{array}{c}\text { \% variation } \\
\text { in natural } \\
\text { frequency }\end{array}$ & $\begin{array}{c}\text { Plate with } \\
\text { two discrete } \\
\text { point masses } \\
\text { (Figure 2(c)) }\end{array}$ & $\begin{array}{c}\text { \% variation } \\
\text { in natural } \\
\text { frequency }\end{array}$ & $\begin{array}{c}\text { Plate with } \\
\text { four discrete } \\
\text { point masses } \\
\text { (Figure 2(d)) }\end{array}$ & $\begin{array}{c}\% \text { variation } \\
\text { in natural } \\
\text { frequency }\end{array}$ \\
\hline 1st & 87 & 73.022 & 16.066 & 84.497 & 2.877 & 83.558 & 3.956 \\
2nd & 193 & 189.87 & 1.621 & 177.85 & 7.849 & 174.55 & 224.63 \\
3rd & 241 & 235.14 & 2.431 & 229.32 & 4.846 & 6.559 \\
4th & 345 & 310.88 & 9.889 & 284.54 & 17.524 & 281.54 & 18.394 \\
\hline
\end{tabular}

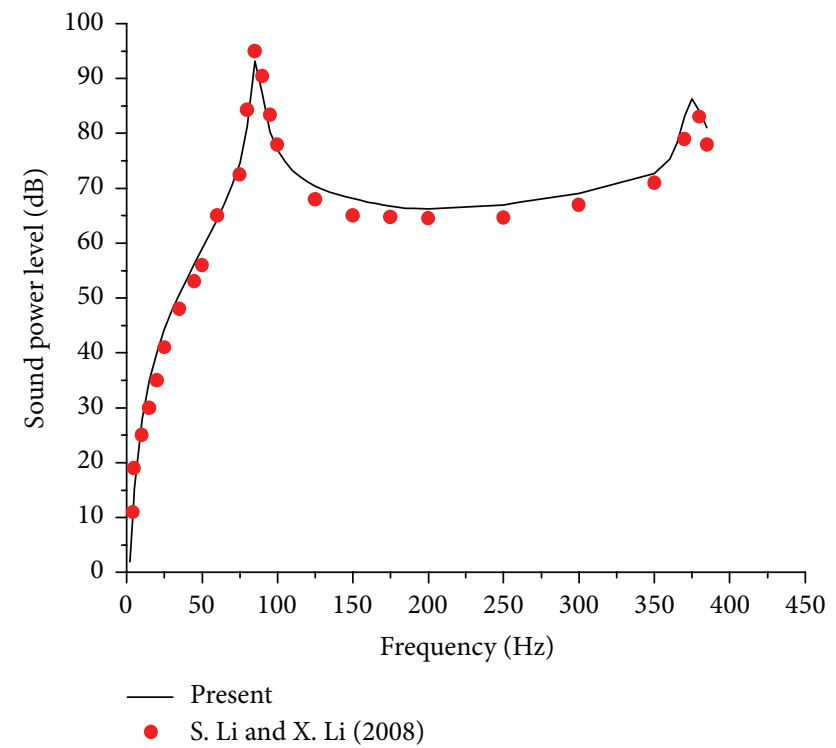

FIgURE 4: Comparison of sound power level with that of S. Li and X. Li [9].

Tables 3 and 4 compare the first four eigenfrequencies $(\omega)$ of the plate of constant thickness $\left(H_{X}=0\right)$ with attached discrete patches and attached discrete point masses, respectively. The decrease in the natural frequency of the plate with attached point masses is more than that of corresponding number of patches. This may be due to contribution of stiffness of the patch to the plate.

Figure 5 compares the eigenfrequency of plate with four discrete patches and four point masses for different modes. It is clear from Figure 5 that eigenfrequency reduction of plate

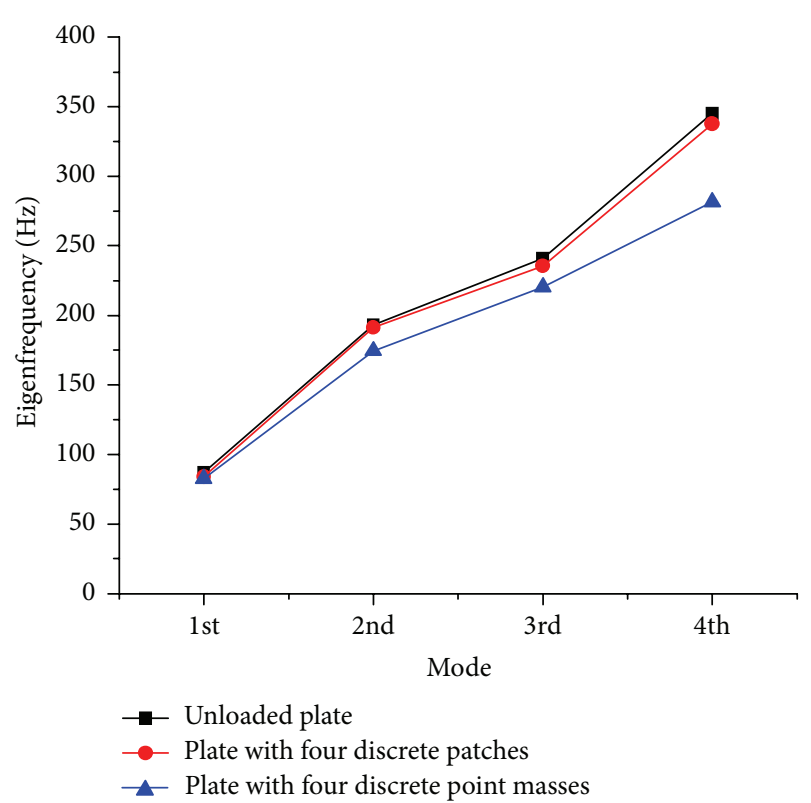

Figure 5: Comparison of the first four natural frequencies.

attached with four discrete point masses is more significant in comparison to plate attached with four discrete patches for 4 th mode. For first natural frequency, effect of point masses and patches is almost the same. Tables 5 and 6 show the effects of varying taper ratio on the natural frequencies of plate with four patches and four point masses, for different cases of thickness variation as shown in Figure 3. It is clear from Table 5 that, with increase in taper ratio, the natural frequencies of plate with four patches for cases 1 and 3 


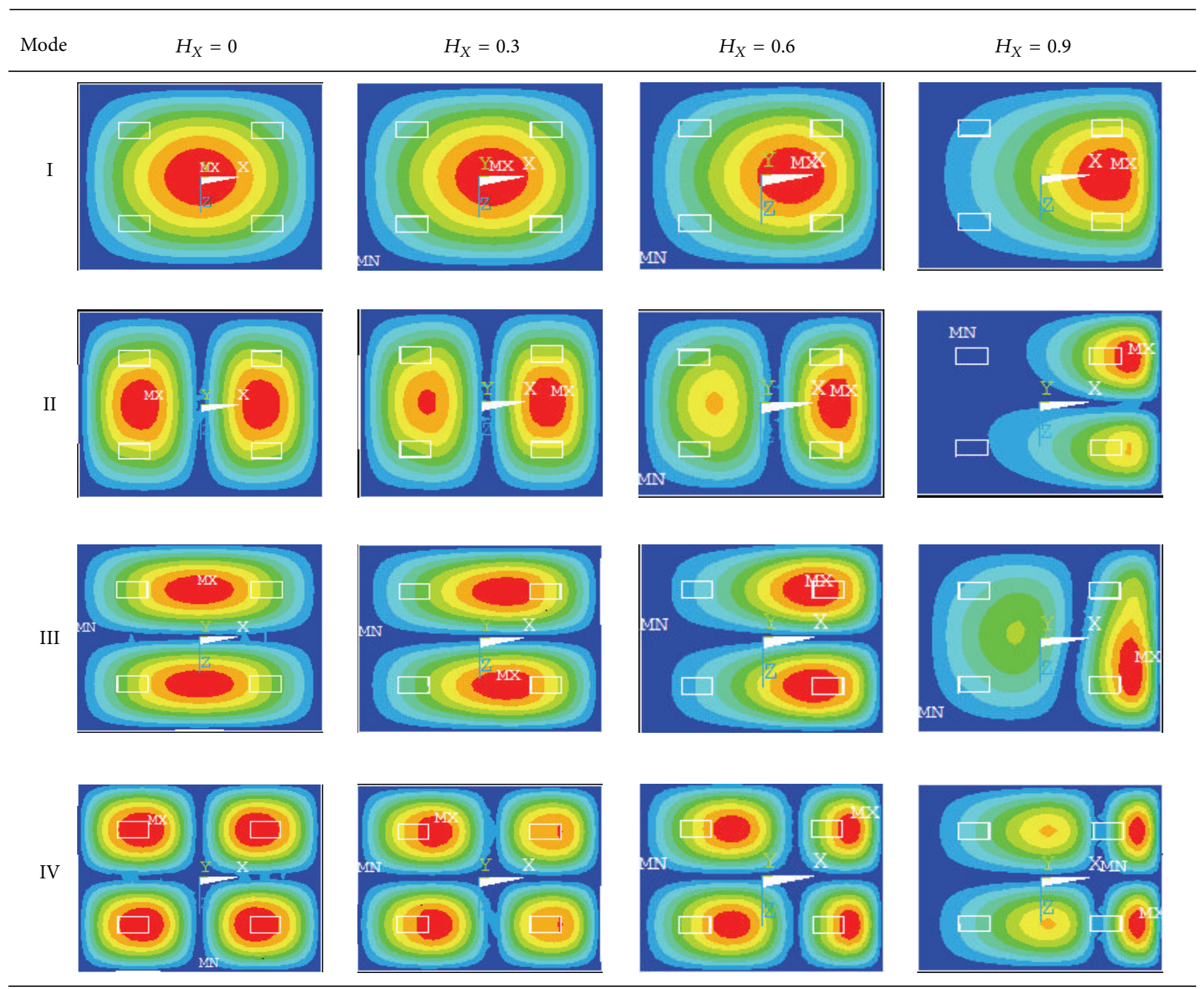

Figure 6: Mode variation of plate with attached four discrete patches with different taper ratios for case 1.

(linearly varying thickness and linearly increasing-decreasing thickness) reduce more in comparison to those of cases 2 and 4 (parabolically varying thickness and parabolically increasing-decreasing thickness), respectively. This is due to higher stiffness contribution with parabolically thickness variation.

However, it is noticed from Table 6 that with increase in taper ratio the natural frequencies of plate with attached four point masses, for cases 1 and 3 (linearly varying thickness and linearly increasing-decreasing thickness), do not always reduce more in comparison to those of cases 2 and 4 (parabolically varying thickness and parabolically increasingdecreasing thickness) due to stiffness modification.

Figures 6 and 7 show the mode shapes of plate with four attached patches and point masses with different taper ratios for case 1, respectively. From Figures 6 and 7, it is observed that different taper ratios $\left(H_{X}=0.3\right.$ and 0.6$)$ do not influence the modal indices of plate with four patches and point masses associated with all the symmetric mode shapes (I, II, III, and
IV). However, when taper ratio is increased to $H_{X}=0.9$, the modal indices associated with 4 th mode get altered.

3.3. Acoustics Response Analysis of Plate with Varying Thickness with Attached Patches/Point Masses with Different Taper Parameter $\left(H_{X}\right)$. The sound power level $(\mathrm{dB}$, reference $=$ $10^{-12} \mathrm{~W}$ ) of simply supported plate with attached point masses and patches with different taper parameter $\left(H_{X}\right)$ is investigated. The plate is excited by a concentrated force of amplitude $1 \mathrm{~N}$ at the center of the plate. A structural damping coefficient of 0.01 is used for plate in air medium. A frequency range of $10-1000 \mathrm{~Hz}$ is chosen in order to investigate the acoustic response analysis for different taper ratios $(0.3,0.6$, and 0.9 ). The patterns of variation of sound power level of plate with attached point masses and patches with different cases are similar in nature. Variation of sound power level with parabolic increasing-decreasing thickness variation of plate with attached "one patch and four patches" and "one point mass and four point masses" is shown in Figures 8-11. 


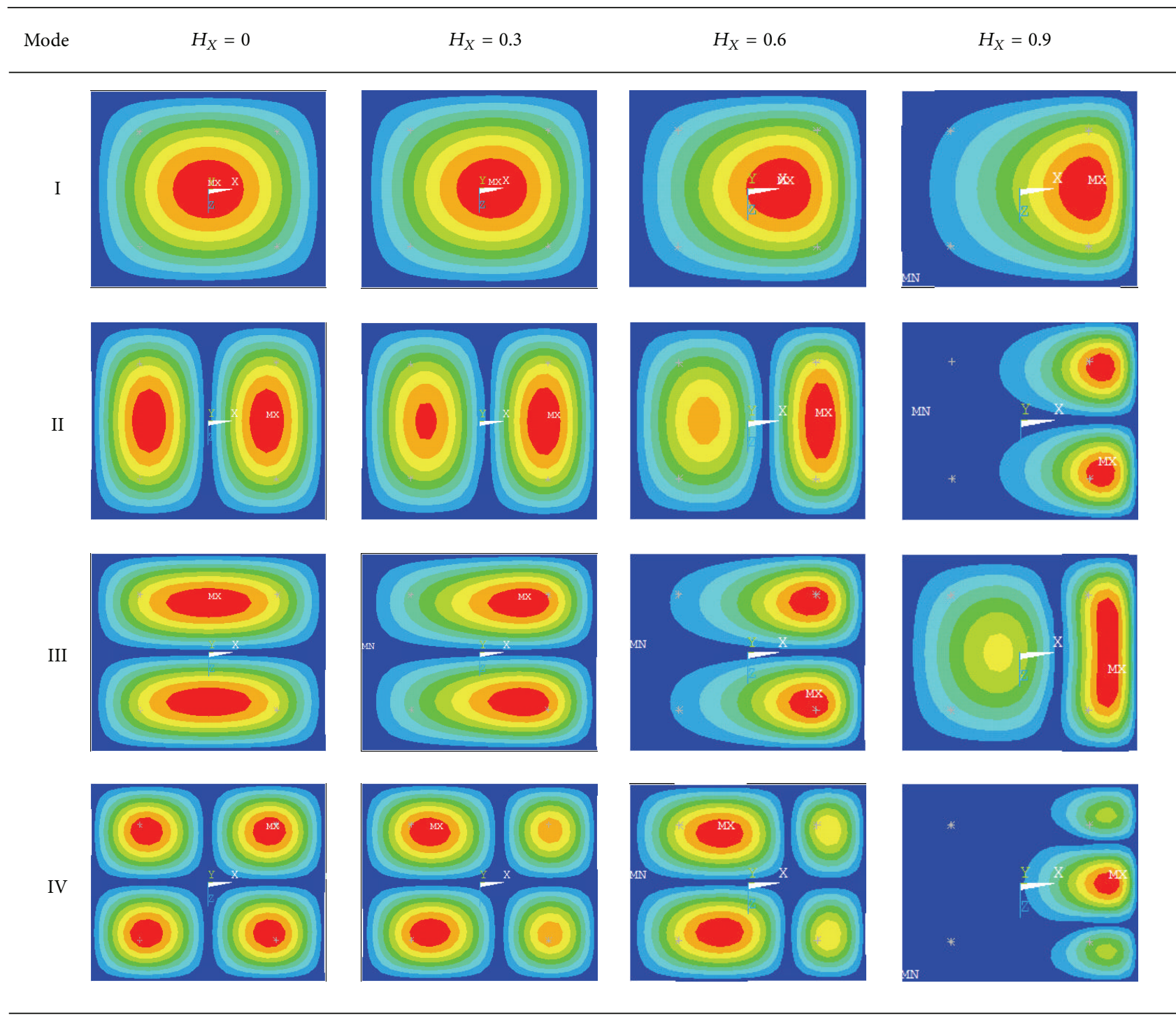

FIGURE 7: Mode variation of plate with attached four discrete point masses with different taper ratios for case 1.

From Figures 8-11, it is obvious that sound power level gets modified by point mass, patch, distribution of point mass/patch, and taper ratio due to stiffness contribution. It is observed that plate with four patches with parabolic increasing-decreasing thickness variation achieves minimum sound power level for a wide range of frequency band at taper ratio $H_{X}=0.6$ as shown in Figure 10. However, it becomes interesting to note that when taper ratio increases to $H_{X}=0.9$, plate with four point masses with parabolic increasing-decreasing thickness variation attains minimum sound power level over a wide frequency range as shown in Figure 11. Further, a comparison of sound power level of plate with four patches at $H_{X}=0.6$ and four point masses at $H_{X}=0.9$ with parabolic increasing-decreasing thickness variation is depicted in Figure 12. It is obvious from Figure 12 that plate with four patches for $H_{X}=0.6$ is more effective in attaining the lower sound power level in wide range of frequency band in comparison to plate with four point masses at $H_{X}=0.9$.

The 10 randomly selected locations of the point on the plate were used to obtain radiation characteristics. The average radiation efficiency of plate with attached one point mass and patch for different taper ratio is also plotted to investigate the effect of taper ratio on sound radiation characteristics as shown in Figures 13 and 14. The slope of average radiation efficiency almost remains the same till the first peak for different taper ratio. To investigate the effect of distribution of point masses and patches at a given taper ratio $\left(H_{X}=0.6\right)$, average radiation efficiency plots are shown in Figures 15 and 16. It is clear that, for low frequency range, the slope of average radiation efficiency almost remains the same, but near first peak, four patches or four point masses cause increase in average radiation efficiency; that is, redistribution of point masses/patches does have effect 
TABLE 5: Comparison of natural frequency variation of plate with attached four patches with different taper ratio $\left(H_{X}\right)$.

\begin{tabular}{lccccc}
\hline \multirow{2}{*}{ Case } & \multirow{2}{*}{ Mode } & \multicolumn{4}{c}{ Natural frequency } \\
& & $H_{X}=0$ & $H_{X}=0.3$ & $H_{X}=0.6$ & $H_{X}=0.9$ \\
\hline \multirow{2}{*}{ I } & I & 85.102 & 83.311 & 82.302 & 78.65 \\
& II & 191.19 & 188.29 & 180.85 & 166.04 \\
& III & 235.43 & 220.02 & 219.96 & 206.45 \\
& IV & 337.57 & 332.58 & 330.42 & 306.92 \\
\hline \multirow{4}{*}{ II } & I & 85.102 & 83.978 & 83.580 & 82.809 \\
& II & 191.19 & 188.99 & 185.83 & 176.58 \\
& III & 235.43 & 230.01 & 229.39 & 209.41 \\
& IV & 337.57 & 335.47 & 332.40 & 325.37 \\
\hline \multirow{4}{*}{ III } & I & 85.102 & 83.571 & 83.571 & 82.894 \\
& II & 191.19 & 189.38 & 187.23 & 169.45 \\
& III & 235.43 & 225.16 & 220.41 & 203.41 \\
& IV & 337.57 & 330.37 & 326.45 & 301.69 \\
\hline \multirow{3}{*}{ IV } & I & 85.102 & 84.84 & 84.79 & 83.581 \\
& II & 191.19 & 190.23 & 188.27 & 174.10 \\
& III & 235.43 & 227.61 & 222.26 & 206.30 \\
& IV & 337.57 & 334.06 & 329.15 & 312.30 \\
\hline \multirow{4}{*}{} & & & & &
\end{tabular}

TABLE 6: Comparison of natural frequency variation of plate with attached four point masses with different taper ratio $\left(H_{X}\right)$.

\begin{tabular}{lccccc}
\hline \multirow{2}{*}{ Case } & \multirow{2}{*}{ Mode } & \multicolumn{4}{c}{ Natural frequency } \\
& & $H_{X}=0$ & $H_{X}=0.3$ & $H_{X}=0.6$ & $H_{X}=0.9$ \\
\hline \multirow{2}{*}{ I } & I & 83.558 & 83.096 & 81.579 & 71.519 \\
& II & 174.55 & 169.98 & 161.80 & 139.18 \\
& III & 224.63 & 219.30 & 196.24 & 152.52 \\
& IV & 281.54 & 272.39 & 267.29 & 255.22 \\
\hline \multirow{4}{*}{ II } & I & 83.558 & 82.27 & 80.301 & 72.754 \\
& II & 174.55 & 170.66 & 167.22 & 159.54 \\
& III & 224.63 & 217.61 & 200.72 & 165.97 \\
& IV & 281.54 & 274.27 & 270.98 & 262.24 \\
\hline \multirow{4}{*}{ III } & I & 83.558 & 83.455 & 82.42 & 79.912 \\
& II & 174.55 & 171.38 & 170.42 & 150.89 \\
& III & 224.63 & 219.32 & 205.04 & 194.90 \\
& IV & 281.54 & 272.21 & 261.36 & 254.90 \\
\hline \multirow{3}{*}{ IV } & I & 83.558 & 83.091 & 82.913 & 81.649 \\
& II & 174.55 & 167.05 & 162.18 & 158.97 \\
& III & 224.63 & 218.05 & 204.16 & 185.41 \\
& IV & 281.54 & 276.36 & 268.62 & 259.12 \\
\hline
\end{tabular}

on radiation efficiency at a given taper ratio after first peak.

3.4. Peak Sound Power Level Variation with Different Taper Ratio for All the Cases. Figures 17(a)-17(f) show the variation of peak sound power level with different taper ratio for plate with patches and point masses for all the cases. It is clear that higher taper ratio of $H_{X}=0.6$ or 0.9 is
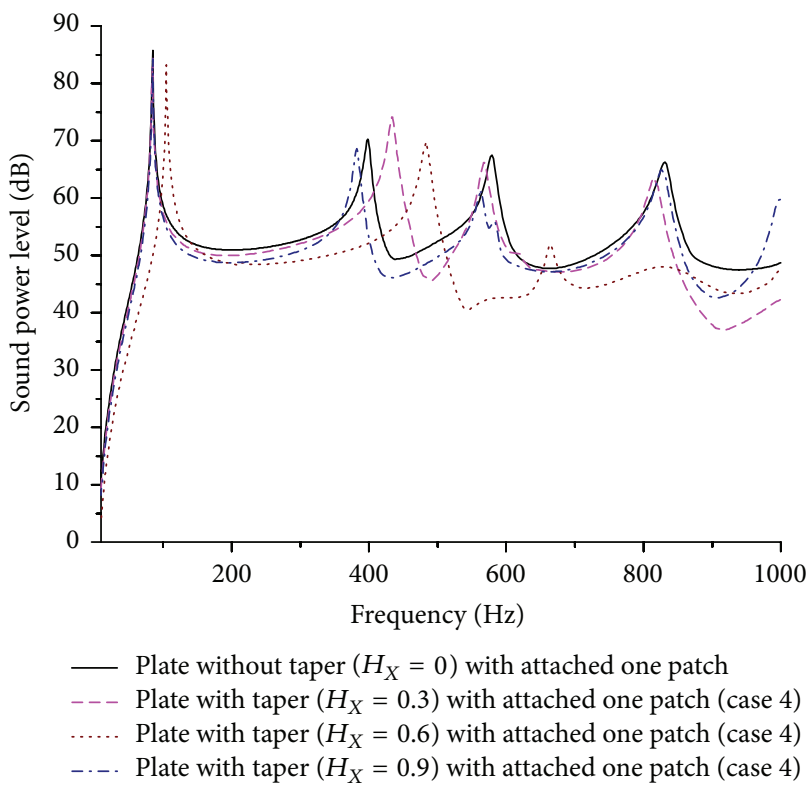

FIgURE 8: Comparison of sound power level $(\mathrm{dB})$ of plate with different tapers $\left(H_{X}=0.0,0.3,0.6\right.$, and 0.9$)$ with attached one patch for case 4 .

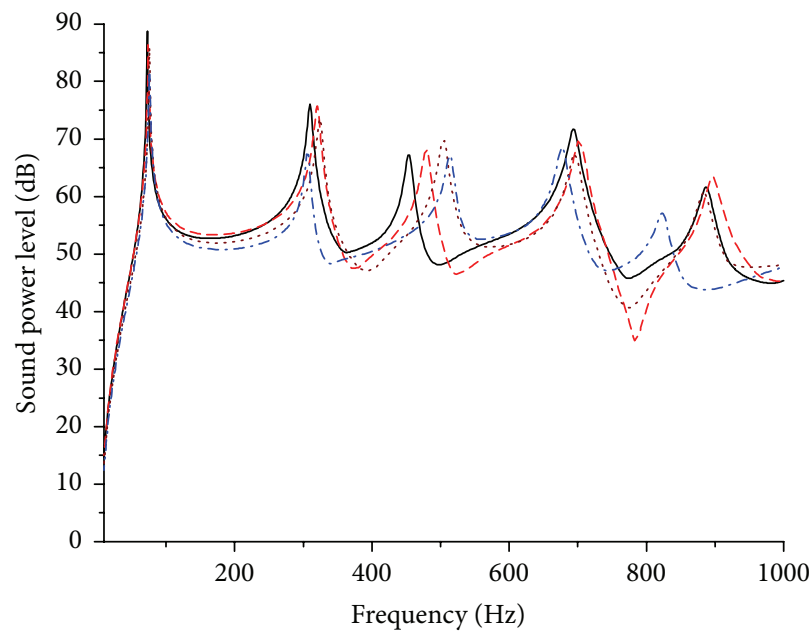

_ Plate without taper $\left(H_{X}=0\right)$ with attached one point mass

- - - Plate with taper $\left(H_{X}=0.3\right)$ with attached one point mass (case 4$)$

..... Plate with taper $\left(H_{X}=0.6\right)$ with attached one point mass (case 4$)$

-.. Plate with taper $\left(H_{X}=0.9\right)$ with attached one point mass (case 4)

Figure 9: Comparison of sound power level $(\mathrm{dB})$ of plate with different tapers $\left(H_{X}=0.0,0.3,0.6\right.$, and 0.9$)$ with attached one point mass for case 4

effective in achieving the minimum peak sound power level with parabolic increasing-decreasing or linearly increasingdecreasing thickness variation. Further, it is noted that the minimum peak sound power level obtained is $78 \mathrm{~dB}$ (Figure $17(\mathrm{e}))$ at $H_{X}=0.6$ with parabolic increasing-decreasing thickness variation for plate with four patches. At $H_{X}=$ 0.6 , parabolically increasing-decreasing thickness variation is most effective in peak sound power level suppression 


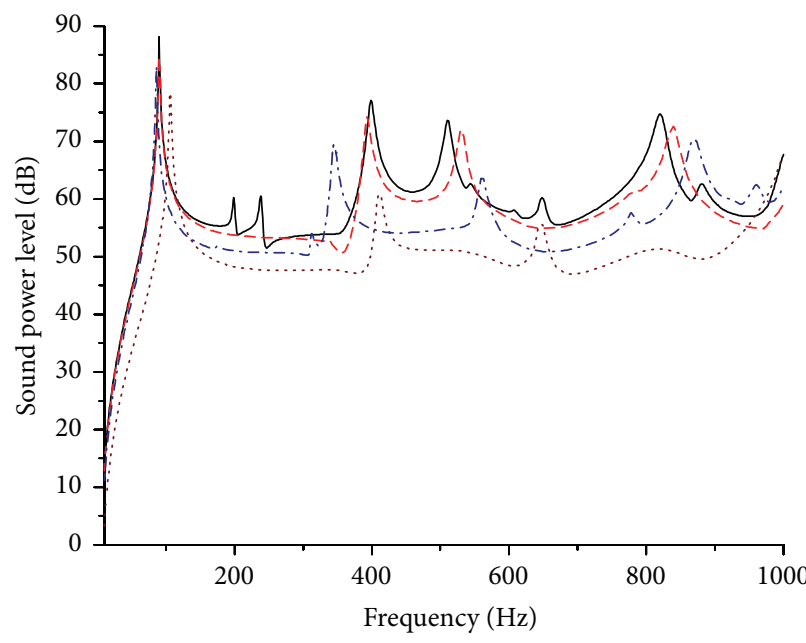

- Plate without taper $\left(H_{X}=0\right)$ with attached four patches

- - Plate with taper $\left(H_{X}=0.3\right)$ with attached four patches (case 4$)$

..... Plate with taper $\left(H_{X}=0.6\right)$ with attached four patches (case 4$)$

-... Plate with taper $\left(H_{X}=0.9\right)$ with attached four patches (case 4$)$

FIGURE 10: Comparison of sound power level $(\mathrm{dB})$ of plate with different tapers $\left(H_{X}=0.0,0.3,0.6\right.$, and 0.9$)$ with attached four patches for case 4 .

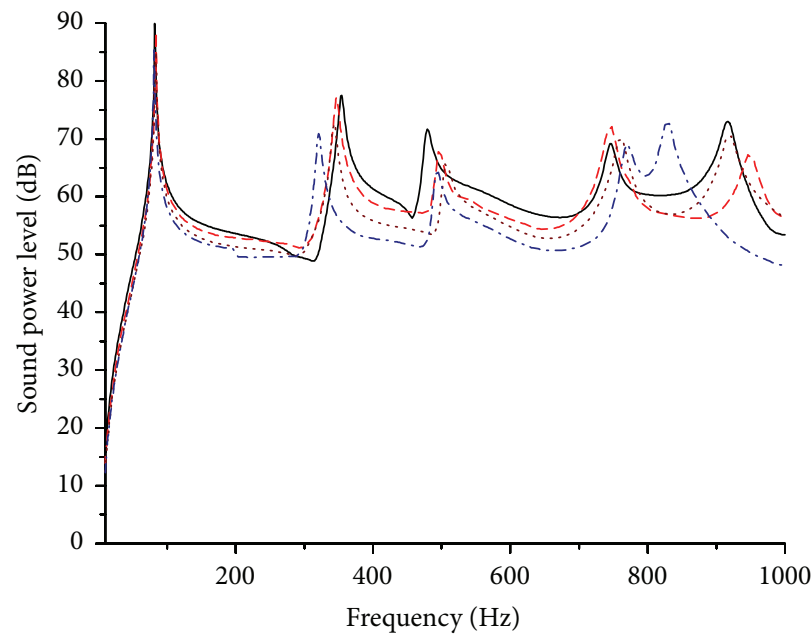

- Plate without taper $\left(H_{X}=0\right)$ with attached four point masses

- - - Plate with taper $\left(H_{X}=0.3\right)$ with attached four point masses (case 4$)$

.... Plate with taper $\left(H_{X}=0.6\right)$ with attached four point masses (case 4 )

-.. Plate with taper $\left(H_{X}=0.9\right)$ with attached four point masses (case 4$)$

Figure 11: Comparison of sound power level $(\mathrm{dB})$ of plate with different tapers $\left(H_{X}=0.0,0.3,0.6\right.$, and 0.9$)$ with attached four point masses for case 4 .

for all the cases considered in this paper. At $H_{X}=0.9$, linearly increasing-decreasing thickness variation is another alternative for peak sound power level suppression.

\section{Conclusions}

A comparison of sound radiation behavior of plate in air medium with attached discrete patches/point masses having

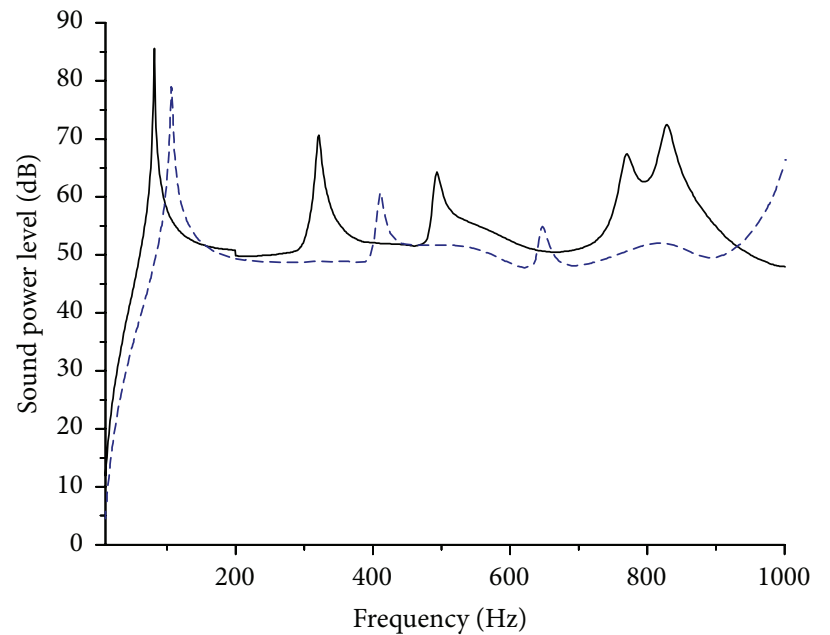

— Plate with taper $\left(H_{X}=0.9\right)$ with attached four point masses (case 4) - - Plate with taper $\left(H_{X}=0.6\right)$ with attached four patches (case 4$)$

Figure 12: Comparison of sound power level $(\mathrm{dB})$ of plate with attached four patches and four point masses at taper ratios $\left(H_{X}=\right.$ $0.9)$ and $\left(H_{X}=0.6\right)$ for case 4 .

different taper ratio is analysed. The masses of the "plate + patches" and "plate + point masses" are kept equal. It has been observed that redistribution of discrete point masses affects the eigenvalues more significantly in comparison to that of patches in eigenvalue suppression. There is significant variation in minimum peak sound power level behavior of plates with parabolic increasing-decreasing thickness variation. It is noticed that higher taper ratio of $H_{X}=0.6$ or 0.9 is effective in achieving the minimum peak sound power level 


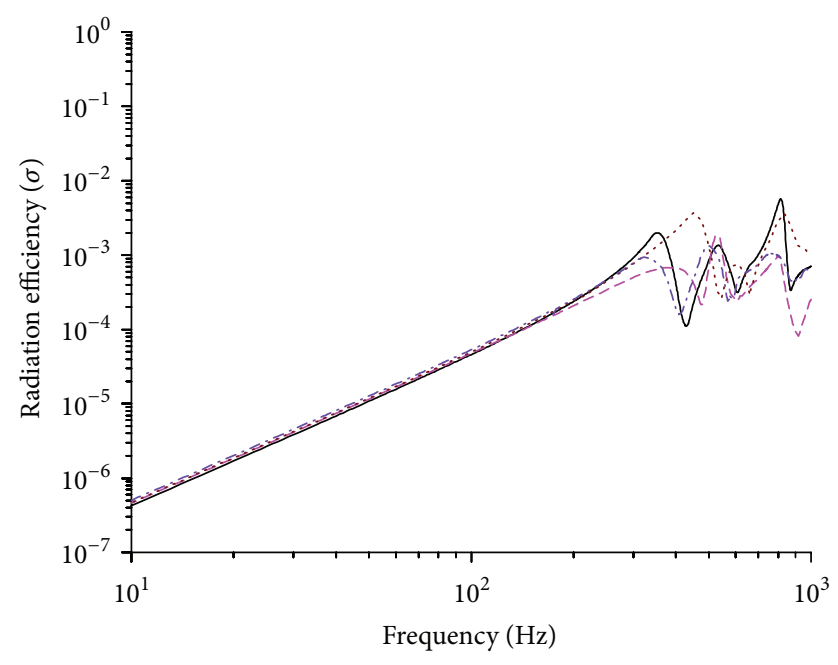

_ Plate without taper with attached one patch

- - Plate with taper $\left(H_{X}=0.3\right)$ with attached one patch (case 4)

..... Plate with taper $\left(H_{X}=0.6\right)$ with attached one patch (case 4$)$

-.. Plate with taper $\left(H_{X}=0.9\right)$ with attached one patch (case 4)

FIGURE 13: Comparison of radiation efficiency of plate with different tapers $\left(H_{X}=0.0,0.3,0.6\right.$, and 0.9$)$ with attached one patch for case 4.

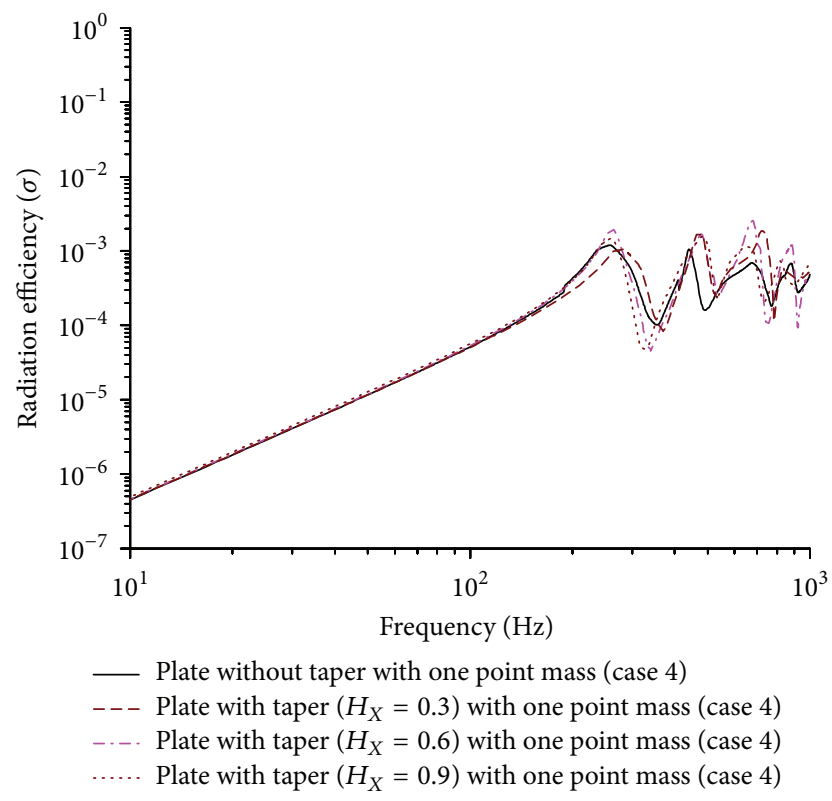

Figure 14: Comparison of radiation efficiency of plate with different tapers $\left(H_{X}=0.0,0.3,0.6\right.$, and 0.9$)$ with attached one point mass for case 4.

with parabolic increasing-decreasing or linearly increasingdecreasing thickness variation. Further, it is noted that the minimum peak sound power level obtained is $78 \mathrm{~dB}$ at $H_{X}=$ 0.6 with parabolic increasing-decreasing thickness variation for plate with four patches at low frequencies. At taper ratio 0.9 , linearly increasing-decreasing thickness variation of plate with patches is another alternative for minimum

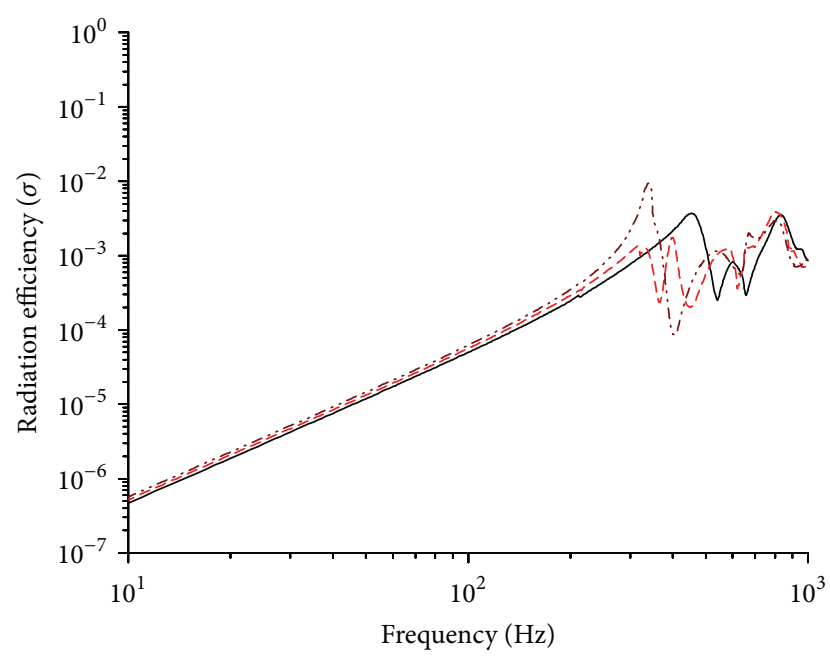

— Plate with taper $\left(H_{X}=0.6\right)$ with attached one patch (case 4)

-- Plate with taper $\left(H_{X}=0.6\right)$ with attached two patches (case 4$)$

.... Plate with taper $\left(H_{X}=0.6\right)$ with attached four patches (case 4$)$

FIGURE 15: Comparison of radiation efficiency of plate with taper $\left(H_{X}=0.6\right)$ with attached one patch, two patches, and four patches for case 4 .

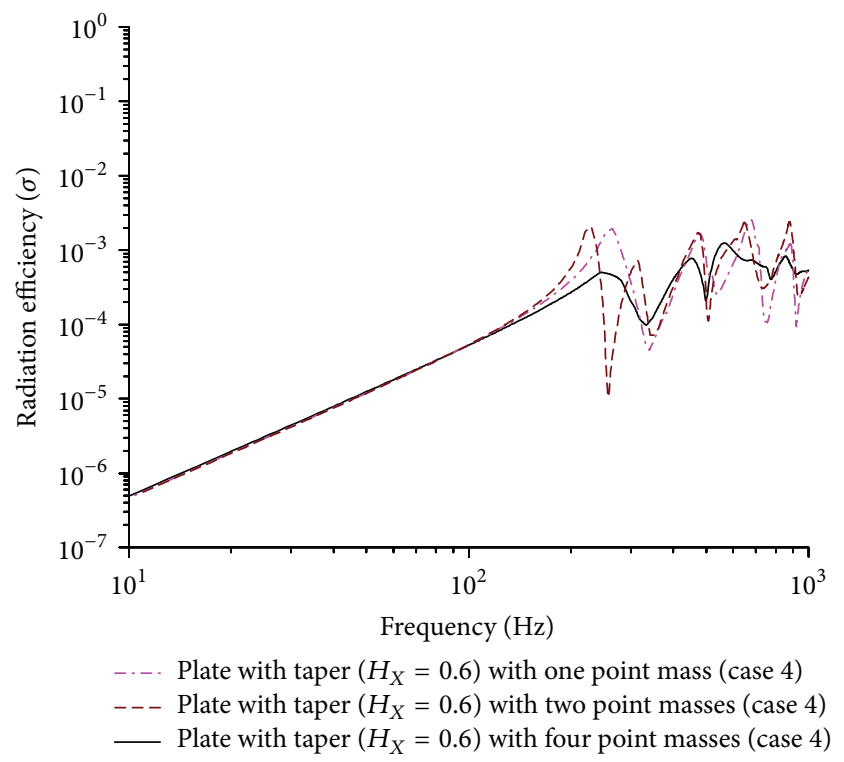

FIGURE 16: Comparison of radiation efficiency of plate with taper $\left(H_{X}=0.6\right)$ with attached one point mass, two point masses, and four point masses for case 4 .

peak sound power level suppression. It is also observed that, for low frequency range, average radiation efficiency almost remains the same for different cases considered, but near first peak, four patches or four point masses cause increase in average radiation efficiency; that is, redistribution of point masses/patches does have effect on average radiation efficiency at a given taper ratio. 


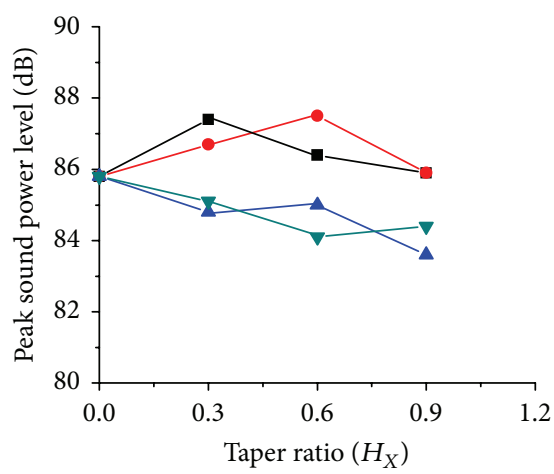

- - Plate attached with one patch (case 1)

-- Plate attached with one patch (case 2)

- $\Delta$ - Plate attached with one patch (case 3)

$-\nabla-$ Plate attached with one patch (case 4 )

(a)

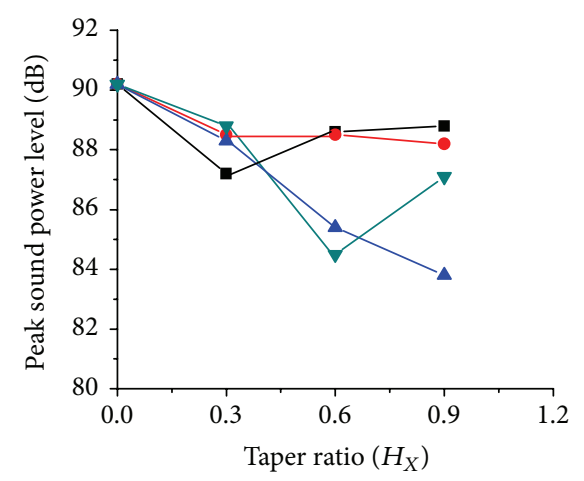

- - Plate attached with two point masses (case 1)

- - Plate attached with two point masses (case 2)

- $\_$- Plate attached with two point masses (case 3)

$-\boldsymbol{\nabla}-$ Plate attached with two point masses (case 4)

(d)

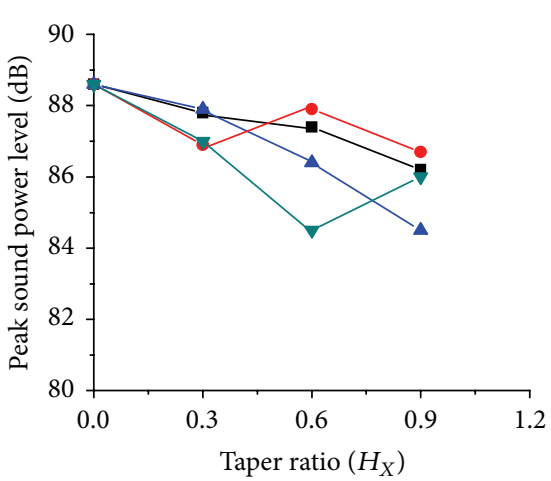

Plate attached with one point mass (case 1) Plate attached with one point mass (case 2)

- - Plate attached with one point mass (case 3)

$-\boldsymbol{-}-$
$-\boldsymbol{\nabla}-$ Plate attached with one point mass (case 4 )

(b)

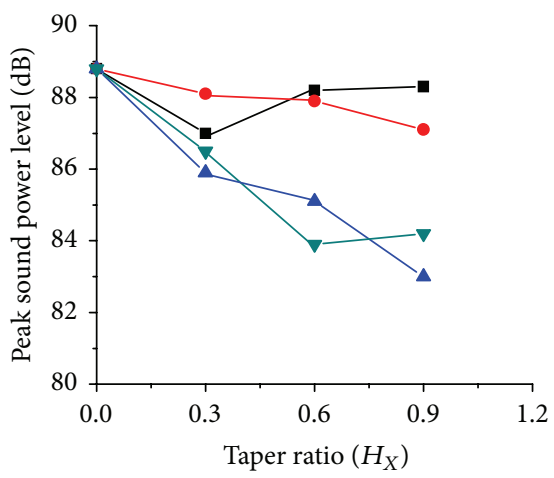

- - Plate attached with two patches (case 1)

-- Plate attached with two patches (case 2)

- $\Delta$ - Plate attached with two patches (case 3)

$-\nabla-$ Plate attached with two patches (case 4)

(c)

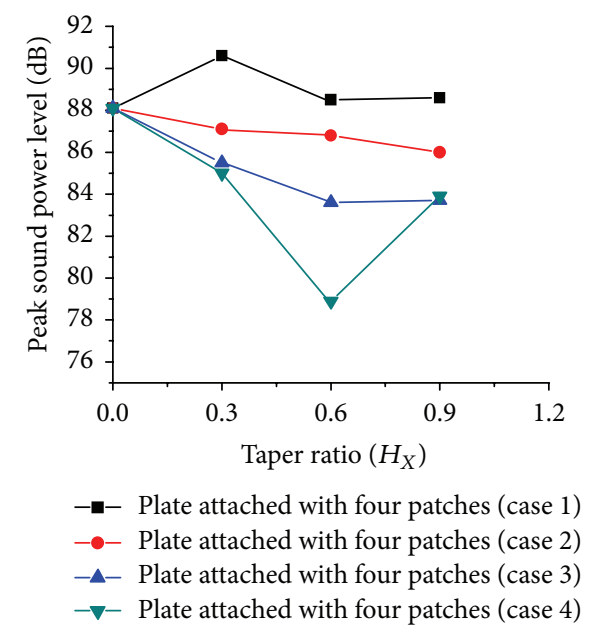

(e)

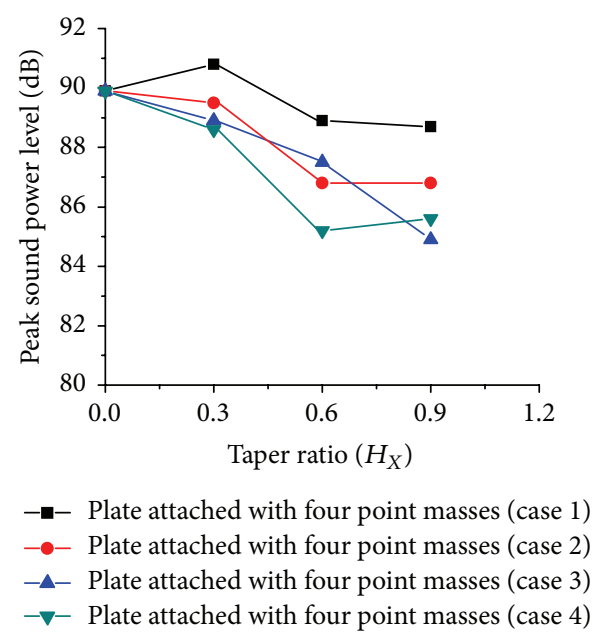

(f)

FIGURE 17: (a) Variation of peak sound power level without taper $\left(H_{X}=0\right)$ and with different taper ratio taken for plate with one patch for cases 1, 2, 3, and 4. (b) Variation of peak sound power level without taper $\left(H_{X}=0\right)$ and with different taper ratio taken for plate with one point mass for cases 1, 2, 3, and 4. (c) Variation of peak sound power level without taper $\left(H_{X}=0\right)$ and with different taper ratio taken for plate with two patches for cases $1,2,3$, and 4 . (d) Variation of peak sound power level without taper $\left(H_{X}=0\right)$ and with different taper ratio taken for plate with two point masses for cases 1,2,3, and 4. (e) Variation of peak sound power level without taper $\left(H_{X}=0\right)$ and with different taper ratio taken for plate with four patches for cases 1, 2, 3, and 4. (f) Variation of peak sound power level without taper $\left(H_{X}=0\right)$ and with different taper ratio taken for plate with four point masses for cases 1, 2, 3, and 4 . 


\section{Conflict of Interests}

The authors declare that there is no conflict of interests regarding the publication of this paper.

\section{References}

[1] F. C. Appl and N. R. Byers, "Fundamental frequency of simply supported rectangular plates with linearly varying thickness," Journal of Applied Mechanics, vol. 32, no. 1, pp. 163-167, 1965.

[2] B. B. Raju, "Vibration of thin elastic plates of linearly variable thickness," International Journal of Mechanical Sciences, vol. 8, no. 2, pp. 89-100, 1966.

[3] J. R. Kuttler and V. G. Sigillito, "Vibrational frequencies of clamped plates of variable thickness," Journal of Sound and Vibration, vol. 86, no. 2, pp. 181-189, 1983.

[4] P. K. Roy and N. Ganesan, "Studies on the dynamic behaviour of a square plate with varying thickness," Journal of Sound and Vibration, vol. 182, no. 3, pp. 355-367, 1995.

[5] M. Huang, X. Q. Ma, T. Sakiyama, H. Matuda, and C. Morita, "Free vibration analysis of orthotropic rectangular plates with variable thickness and general boundary conditions," Journal of Sound and Vibration, vol. 288, no. 4-5, pp. 931-955, 2005.

[6] G. Petrone, V. D’Alessandro, F. Franco, and S. de Rosa, "Numerical and experimental investigations on the acoustic power radiated by Aluminium Foam Sandwich panels," Composite Structures, vol. 118, no. 1, pp. 170-177, 2014.

[7] F. Franco, S. De Rosa, and T. Polito, "Finite element investigations on the vibroacoustic performance of plane plates with random stiffness," Mechanics of Advanced Materials and Structures, vol. 18, no. 7, pp. 484-497, 2011.

[8] G. Altintas, "Effect of mass based imperfections on behavior of linear vibrating plates near Degenerate Modes," Journal of Vibration and Control, vol. 15, no. 2, pp. 219-231, 2009.

[9] S. Li and X. Li, "The effects of distributed masses on acoustic radiation behavior of plates," Applied Acoustics, vol. 69, no. 3, pp. 272-279, 2008.

[10] A. Putra and D. J. Thompson, "Sound radiation from rectangular baffled and unbaffled plates," Applied Acoustics, vol. 71, no. 12, pp. 1113-1125, 2010.

[11] P. Jeyaraj, "Vibro-acoustic behavior of an isotropic plate with arbitrarily varying thickness," European Journal of Mechanics, A/Solids, vol. 29, no. 6, pp. 1088-1094, 2010.

[12] H. Nelisse, O. Beslin, and J. Nicolas, "A generalized approach for the acoustic radiation from a baffled or unbaffled plate with arbitrary boundary conditions, immersed in a light or heavy fluid," Journal of Sound and Vibration, vol. 211, no. 2, pp. 207225, 1998.

[13] N. Atalla, J. Nicolas, and C. Gauthier, "Acoustic radiation of an unbaffled vibrating plate with general elastic boundary conditions," Journal of the Acoustical Society of America, vol. 99, no. 3, pp. 1484-1494, 1996.

[14] D. J. Thompson, G. Xie, and C. J. C. Jones, "The radiation efficiency of baffled plates and strips," Journal of Sound and Vibration, vol. 280, no. 1-2, pp. 181-209, 2005.

[15] P. K. Roy and N. Ganesan, "Studies on the response of a square plate with variable thickness excited by uniformly distributed load," Computers and Structures, vol. 45, no. 3, pp. 593-603, 1992.

[16] L. Rayleigh, The Theory of Sound, Macmillan, London, UK, 2nd edition, 1896.
[17] F. J. Fahy and P. Gardonio, Sound and Structural Vibration: Radiation, Trans-Mission and Response, Academic Press, London, UK, 2nd edition, 2006.

[18] L. Cremer, M. Heckl, and B. A. T. Petersson, Structure-Borne Sound, Springer, Berlin, Germany, 3rd edition, 2005.

[19] C. E. Wallace, "Radiation resistance of a rectangular panel," Journal of the Acoustical Society of America, vol. 51, no. 3, pp. 946-952, 1972. 


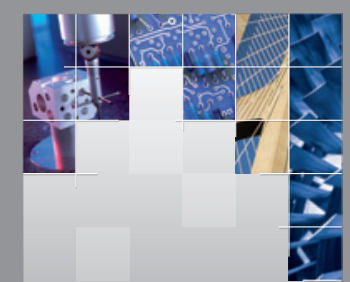

\section{Enfincering}
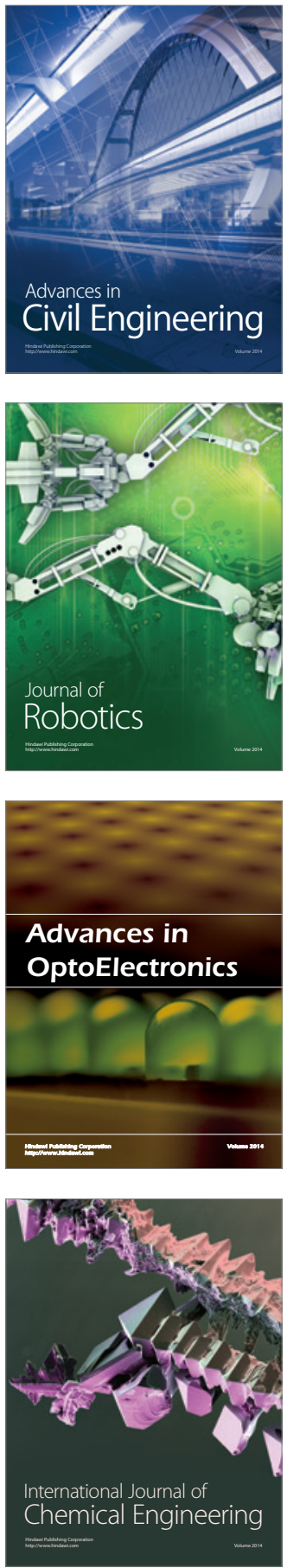

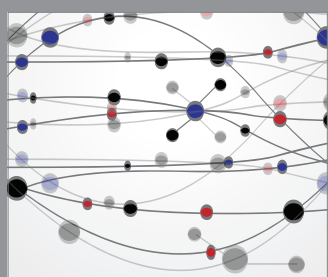

The Scientific World Journal

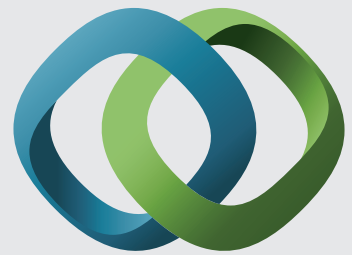

\section{Hindawi}

Submit your manuscripts at

http://www.hindawi.com
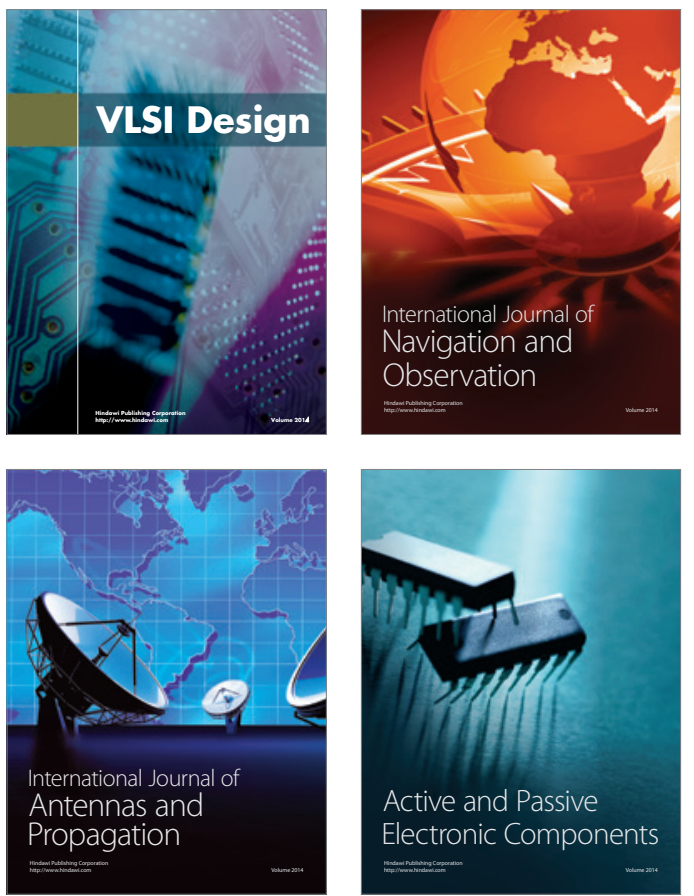
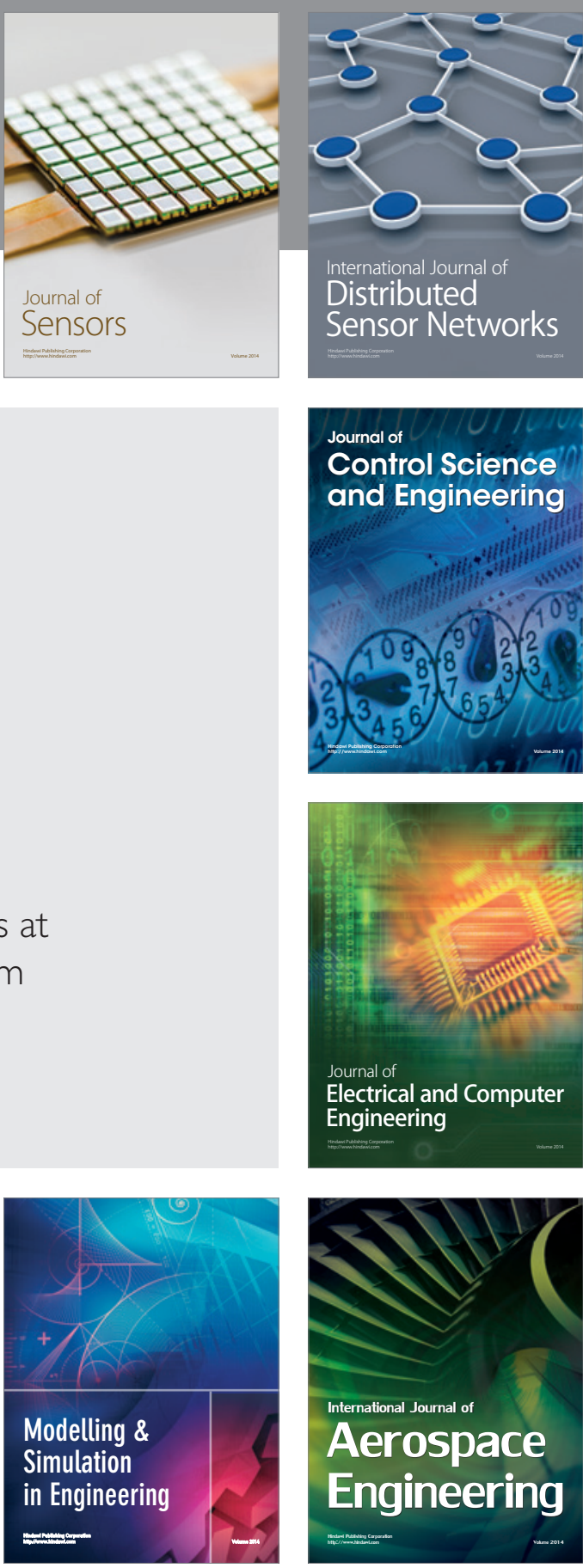

International Journal of

Distributed

Sensor Networks

Journal of

Control Science

and Engineering
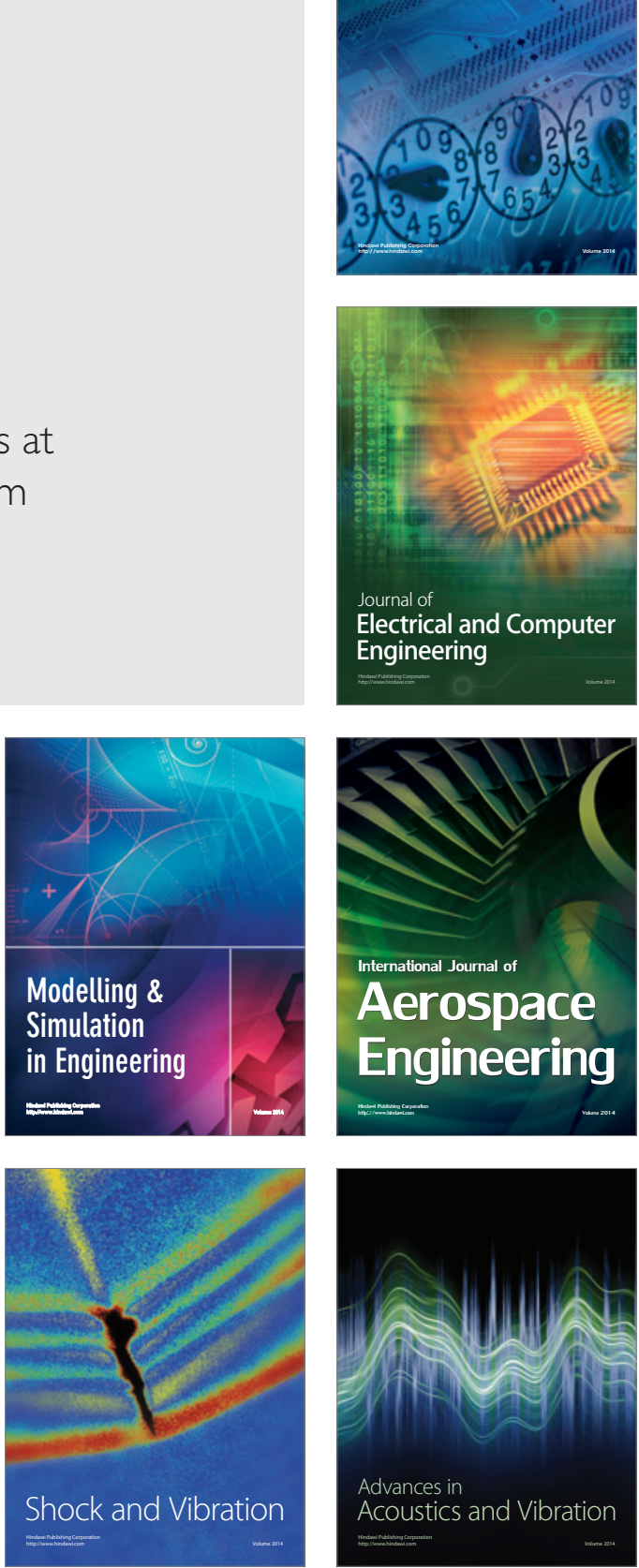